\title{
Professor Manindra Chandra Chaki (1913 - 2007): A Legendary Indian Geometrician
}

\author{
Sanatan Koley* \\ Former Headmaster, Jagacha High School (H.S.), Howrah.
}

(Received 31 October 2020; revised 17 December 2020)

\begin{abstract}
Manindra Chandra Chaki, former Sir Asutosh Birth Centenary Professor of Higher Mathematics (formerly Hardinge Professor) and Head of the Department of Pure Mathematics, University of Calcutta, was a prolific researcher in various branches of Geometry. He made notable contributions in the fields of Classical and Modern Differential Geometry, History of Mathematics, etc. His contributions, published in reputed national and international journals earned him great recognition from the international community of geometers. Besides, the notion of pseudo symmetric manifold, as introduced by Professor Chaki in 1987, is so innovative that, it is now referred to as Chaki manifold, denoted by Chaki $(P S)_{n}$ in mathematical literature. As a tribute to the towering geometer of India, an attempt has been made in this article to highlight some of his memorable and fundamental research contributions in several fields.
\end{abstract}

Key words: A. G. Walker, Chaki manifold, Differential Geometry, H. S. Ruse, K. Yano, L. P. Eisenhart, M. Toomanian, Quasi Einstein manifolds, R. N. Sen, T. J. Willmore, University of Calcutta.

\section{Early life and education}

Professor Manindra Chandra Chaki, well known as M. C. Chaki, was born on 1st July, 1913 at the village Deuli, Bagura district in undivided Bengal, now in Bangladesh (Figure 1). His father Keshab Chandra Chaki was a dignified landlord and mother, Kunjakamini Devi, was a very devoted and pious lady. He received his early education in different institutes in undivided Bengal. It is to be mentioned here that in his early school days he did not find much interest in Mathematics; but his private tutor Sri Durgadas Banerjee motivated him to develop it.

After completing his Matriculation examination from Gaibandha High School in the district of Rangpur, in 1930 in 1st division with star mark, he came to Calcutta and got

DOI: $10.16943 / \mathrm{ijhs} / 2020 / \mathrm{v55i4/158290}$

*Email: sanatankoley2017@gmail.com.

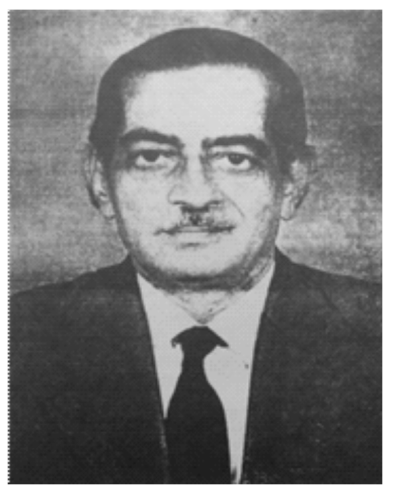

Figure 1 Professor M. C. Chaki (1st July 1913-21st July 2007).

himself admitted to Bangabasi College, a private college in the city. In 1932, he passed I.Sc. examination from this college in 1st division. Thereafter, he was admitted to Ra- 
jshahi Government College in B.A. class with Honours in English. Ultimately, as advised by B. M. Sen (1888-1978), the then Principal of the college, he decided to join the Honours classes in Mathematics (Chaki 1991, p. 77). He obtained B.A. degree from this college in 1934 with second class Honours in Mathematics and with Sanskrit as a subsidiary subject. Needful to say that, besides Mathematics, Sanskrit was his second favourite subject which he cultured throughout his life.

He obtained M.A. degree in Pure Mathematics from Calcutta University in 1936 occupying second position in 1st class. After obtaining M.A. degree, he joined Law classes at his uncle's advice but did not find any interest in this subject and left it. In 1956, he obtained Ph.D. in Pure Mathematics from the University of Calcutta for his thesis entitled 'On some problems in Riemannian Geometry'. The thesis was written under the guidance of R. N. Sen (1896-1974), the then Head and at the same time Hardinge Professor in the Department of Pure Mathematics. This work was highly praised by L. P. Eisenhart (18761965) of the Princeton University, U.S.A., who was one of the examiners of the thesis (Mukherji and Bhattacharjee 2014, p. 90).

\section{Teaching career}

Professor Chaki joined the Azizul Haque College in Bagura, as a lecturer in Mathematics and English during the period 1939-1945. He next joined Bangabasi College in Calcutta as a lecturer in Mathematics and was in service there from 1945 to 1952 . He was a part-time lecturer in the Department of Pure Mathematics, University of Calcutta, during 1950-1952 and in 1952, he joined there as a full-time lecturer. He taught there as a Reader during 1960-1972. In 1972, he was selected as Sir Asutosh Birth Centenary Professor of Higher Mathematics (formerly Hardinge Professor) in Calcutta University. It may be noted that Professor Chaki was the first person to hold this most prestigious Chair. In 1974, he became Head of the Department of Pure Mathematics. Chaki adorned these two prestigious posts simultaneously till his retirement in 1978 from the University service.

As a teacher, Professor Chaki was very successful and popular among the students. He was well conversant with languages like French, German, Russian and Italian which helped him to go through the research papers pub- lished in those languages. This enriched his mathematical and geometric knowledge, thus giving him impetus to carry on further research. A lot of students have been awarded Ph.D. degree under his guidance. Amongst them Bandana Barua, A. N. Raychowdhury, Asoke Kr. Ray, Dipak Kr. Ghosh, A. K. Bag (History of Mathematics), P. K. Majumdar (History of Mathematics), K. K. Sharma, U. C. De, M. Tarafdar, A. Konar and Balai Chaki deserve special mention for their valuable contributions. Later, Bandana Barua, Dipak Kr. Ghosh, U. C. De and M. Tarafdar served as faculty members in the Department of Pure Mathematics, Calcutta University and also did good research works in many areas of Geometry. A. N. Raychowdhury became Professor of Mathematics, NIT, Durgapur, Deemed University; Asoke Kr. Roy, K. K. Sharma and A. Konar became Professor of Mathematics in Jadavpur, Tripura and Kalyani University respectively. A. K. Bag took the responsibility of Indian Journal of History of Science as its Editor. P. K. Majumdar was Professor of Indian Astronomy in the School of Vedic Studies at Rabindra Bharati University, and Balai Chaki worked as a scientist in CSIR, Government of India. Some other students who have been awarded Ph.D. under Professor Chaki's supervision are: Gouri Kumar, S. K. Kar, S. K. Saha, M. L. Ghosh, S. P. Mondal, Sanatan Koley, Sarbari Ray-Guha, R. K. Maity, P. K. Ghoshal, Mahua Banerjee, Satyabachi Sar (Pedagogic Mathematics),... all of them proved to be successful teachers in their later lives.

\section{Teaching contributions}

In the early period of his teaching life in the Department of Pure Mathematics, Professor Chaki started taking classes in various difficult subjects like Projective Geometry, Non-Euclidean Geometry, etc. His way of teaching was exceptionally good and students took much interest in these difficult subjects. It was so attractive that students from other universities used to come to the Department of Pure Mathematics to attend his classes. Even when he was taking undergraduate classes in Bangabasi College, students from other nearby colleges flocked there to listen to his lectures.

Professor Chaki always encouraged junior teachers as well as students to learn new subjects. As a result topics like Geometry of Differentiable manifolds, Mechanics on manifolds, Special and General Theory of Relativ- 
ity, etc. were introduced in the curricula of post-graduate classes. He was always interested in applying his theories in other branches of sciences. He wrote valuable articles on Statistics, Anthropology, etc. Some of his research students obtained Ph.D. degree in Pedagogic Mathematics (Satyabachi Sar) and History of Mathematics (A. K. Bag, P. K. Majumdar).

\section{Research contributions}

Professor Chaki was a prolific researcher in various branches of Geometry. He made notable contributions in the fields of Classical Differential Geometry, Differential Geometry of Manifolds, General Theory of Relativity and Cosmology. He wrote articles on Mathematical Biology, Mathematical Anthropology, Pedagogic Mathematics, History of Mathematics. Nearly 25 students obtained Ph.D. degree under his supervision and more than 70 research papers of high quality have been published in various reputed national and international Journals.

Professor Chaki had great interest in teaching Mathematics through mother tongue. For this reason, he took initiative to publish a glossary of Mathematical terms. Under his Chairmanship, a comprehensive glossary of Mathematical terms came out (in two volumes), published by the West Bengal State Book Board. He wrote some text books on Mathematics starting from the Higher Secondary level to Postgraduate level and also biographical memoirs of a few eminent Fellows of the Indian National Science Academy (INSA), namely: R. N. Sen, B. M. Sen, Jotirmoy Ghosh and N. L. Ghosh (MCCBCCV, 2012, pp. 18-19). Moreover, he wrote biography of Syamadas Mukhopadhyay.

\section{A few notable research papers}

A few important research papers published by Professor Chaki are discussed here in a nutshell. The title of the paper that should be mentioned first is 'On a non-symmetric Harmonic Space' (BCMS, 1952, pp. 37-40). In this paper Professor Chaki constructed an example of a simple harmonic space of dimension $n, n>4$, which is neither flat nor symmetric in the sense of Cartan. This nullifies the conjecture made by A. Lichnero-wicz (1915-1998), a noted French differential geometer, that the dimension of a harmonic space must be less than or equal to 4 ; otherwise it will become a Cartan-symmetric space. The aforecited paper (of Professor Chaki) has been internationally acclaimed. Relevantly, this paper has been referred to in the book entitled 'Harmonic Spaces' by H. S. Ruse (19051974), A. G. Walker (1909-2001) and T. J. Willmore (19192005), published in 1961. Thereafter a large number of research papers had been published in peer reviewed national and international Journals.

In 1963, Professor Chaki, in collaboration with his first research student B. Gupta (Barua) introduced the notion of a new type of Riemannian space called Conformally Symmetric space which is defined as follows:
An n-dimensional Riemannian space $V_{n}(\mathrm{n}>3)$ is called a Conformally Symmetric space, if the conformal curvature tensor $C^{h}{ }_{i j k}$ satisfies the condition $C^{h}{ }_{i j k, l}=0$ where comma denotes co- variant differentiation with respect to the met- ric tensor $g_{i j}$ of $V_{n}$ (IJM, 1963, pp. 113-122). Two interesting and important results proved there, are i) 'A conformally symmetric space is of constant scalar curvature if and only if the first covariant derivative of Ricci tensor is a symmetric tensor.' ii) 'Every Einstein con- formally symmetric space is symmetric in the sense of Cartan.'

This paper had great impact on the research workers on Differential Geometry. The Polish and the Japanese schools which are the most active schools in Differential Geometry appreciated the work and published a large number of papers on this type of space. Later, it has been found to be useful in General Theory of Relativity. Some important contributions have been made on such manifolds by research workers in several countries like U.S.A., U.K., Canada, Russia, Belgium etc. (Guha 2003, p.779). T. J. Suh of Kyungpook National University, South Korea, R. Sharma of Michigon State University, published very important papers based on conformally symmetric semiRiemannian spaces and conformally symmetric spacetime, which appeared in Rocky Mountain Journal of Mathematics, 2005, and Journal of Mathematical Physics, 1999, respectively.

Professor Chaki started his research work on Riemannian geometry using Eisenhart's index method to represent a tensor. But in early 1980, he became interested in 
global differential geometry of manifolds and started writing his research papers using modern index-free notations and encouraged his students to do so.

In 1987, Professor Chaki introduced the idea of pseudo symmetric structures on manifolds (ASAUAICDI 1987, pp. 53- 58). In mathematical literature, this has been referred to as Chaki Manifold by M. Toomanian (Mathematical Reviews, U.S.A., September, 1993, p. 4970). An n-dimensional Chaki manifold is nowadays denoted by Chaki $(P S)_{n}$. Professor Chaki dedicated this paper to famous geometrician Kentaro Yano (1912-1993) on his 74th birthday. Professor Yano was very happy to get it and praised it very much. In this paper, Professor Chaki obtained six important theorems among which four are as follows:

1. 'In a $(P S)_{n}$ the associated 1-form is closed.'

2. 'An Einstein $(P S)_{n}(n>2)$ is of zero scalar curvature.'

3. 'If in a $(P S)_{n}(n>3)$ the Ricci tensor is a Codazzi tensor, then its conformal curvature tensor is conservative.'

4. 'If in a $(P S)_{3}$ the Ricci tensor is a Codazzi tensor, then the manifold is conformally flat.'

Introduction of the idea of pseudo symmetric structures on a manifold is one of Professor Chaki's best contributions in the field of geometry of differentiable manifolds. This concept has been generalized by several research workers in many ways. Mainly Hungarian, Indian, Turkish and Yugoslav researchers published large number of papers in this area in international Journals (MCCBCCV 2012, p. 79). Using the basic space as a $(P S)_{n}$ many important and interesting results are obtained in General Relativity and Cosmology. A paper of Professor Chaki (with one of his research students S. Ray) dealing with such a relativistic space-time has already been published in the International Journal of Theoretical Physics in the year 1996 (IJTP, 1996, pp. 1027-1032).

In the year 2000, Professor Chaki (with his research student R. K. Maity) introduced the notion of quasi Einstein manifold (PMD, 2000, pp. 297-306). This paper is a consequence of Professor Chaki's continuous research on relativistic manifolds. In this paper, he defined a quasi Einstein manifold by putting a restriction on the Ricci tensor as, $S(Y, Z)=a g(Y, Z)+b A(Y) A(Z)$, where $a, b(\neq 0)$ are scalars and $A, a$ differentiable 1-form.

An n-dimensional manifold with this type of structure is denoted by $(Q E) n$. Later he gave a few generalizations of the above mentioned idea. All these ideas have been proved to be very useful in the study of relativistic fluid space-time. Researchers of today are much benefitted by this structure.

In 2006, when Professor Chaki was 93 years old, he introduced the concept of an almost pseudo Ricci symmetric manifold. It was a generalization of the notion of pseudo Ricci symmetric manifold. An n-dimensional manifold of this kind is denoted by $A(P R S)_{n}$. In the same year Professor Chaki wrote his last scientific paper based on this new concept jointly with famous mathematician T. Kawaguchi of Japan. This paper was published posthumously in 2007 in the Journal Tensor, N.S. (2007, pp.1014). In this connection a remark made by Dr. B. Barua in a paper published in 2013 may be quoted. She wrote: "This is a unique example! It shows how powerful his creative mind was and how much dedicated he was to his subject of interest!" (Tensor, N.S., 74, 2013: 212-215). Since Professor Chaki could write papers at the ripe age of 94 , he claimed that he was the senior most active mathematician at his time. In the afore-said paper they (Chaki and Kawaguchi) obtained the following important theorems of a conformally flat $A(P R S)_{n}(n>3)$.

i) 'The scalar curvature of a conformally flat $A(P R S)_{n}(n>3)$ can not be zero.' ii) 'A simply connected conformally flat $A(P R S)_{n}(n>3)$ of non-zero constant scalar curvature can be isometrically immersed in the Euclidean manifold $E_{n+1}$ as a hypersurface.'

\section{Honours and distinctions}

Professor Chaki received many honours from numerous academic institutions and was associated with various learned societies and associations. In 1951, he was elected a fellow of the Royal Astronomical Society, London. Since 1964, he had been working as a Reviewer of Mathematical Reviews, U.S.A. till his death. He was elected the President of the section of Mathematics and Statistics of Indian Science Congress Association for its 63rd session held at Visakhapatnam in 1976. He was the President of the Calcutta Mathematical Society for the year 1981. In 1984, he was appointed as Research Associate in Indian National 
Science Academy (INSA), New Delhi. There he submitted a report on the 'History of development of geometry in India during 19-20th century'. In 1993, he was awarded the honour of 'Teacher of Eminence' by the University of Calcutta. In 1998, the name of Professor Chaki was included in the Board of Editors of the internationally reputed Journal Tensor, Japan. He was an Honorary fellow of the Asiatic Society, Calcutta.

It is remarkable that even at the age of 94, Professor Chaki continued to guide researchers, inspired students of mathematics and mathematical sciences, and participated in various activities meant to widen the scope of mathematics and its applications. He delivered invited lectures in several Indian Universities and Institutes among which a few are mentioned below.

1. Karnatak University, Dharwad - Mathematical Biology, 1977.

2. Mehta Research Institute, Allahabad - Modern Differential Geometry and Biology, 1978.

3. Shivaji University, Kolhapur, Maharashtra - Einstein’s Scientific Philosophy, 1979.

4. Allahabad University - B. N. Prasad Memorial Lecture, 1991.

5. Indian Statistical Institute (ISI), CalcuttaApplication of Differential Geometry to Atmospheric science, 1992.

6. Platinum Jubilee lecture of Indian Science Congress Association (1994-1995) - The role of modern differential geometry on Physical Science and Statistics.

7. Delhi University - Differential Biology, 1995.

8. Benaras Hindu University-The nature and cultural significance of mathematics, 1997.

\section{Personal life}

Professor Chaki was a bachelor. He spent the major part of his life in a hotel in Calcutta. After the untimely death of his elder brother he had to look after his family residing at Jhargram. He had a wonderful quality of recognizing his acquaintances even if he had met them after a long time. Specially, in case of his students - old and new, he could at once remember his name, session, specialities etc. For this special quality, he was said to have a photographic memory. It is noteworthy that apart from various branches of Geometry, Professor Chaki took interest in writing articles on some topics on social life. Some of these are:

1. The Chhau dance of Seraikela, The Modern student, December, 1938.

2. The Gold standard - its theoretical aspects, The Modern Student, July, 1939.

3. On the Indian Zodiac, J. AASM Biophys. Res. Cent. Inaugural Volume, 1987, pp. 1-7.

4. Axiomatic Kinship Theory: (with B. Chaki), SYRI, 1.1 (1990): 3-8.

He took much interest in Indian Classical Music. He had good relationship with well-known musicians like Ustad Vishmadev Chattopadhyay, Sukhendu Goswami, Santosh Sengupta, Sachin Dev Burman and some others. He himself was a good singer. He passed away on 21st July, 2007 at the age of 94+ years. The internationally famous legendary geometrician, Professor Chaki is now survived by his innumerable students, grand students and admirers in the country and abroad.

\section{M. C. Chaki Centre}

M. C. Chaki Centre for Mathematics and Mathematical Sciences was set up in Calcutta on 13th July, 1996 by the students, colleagues and admirers of Professor Chaki for advancement of mathematics teaching, learning and research. It was named after him as a mark of respect. The centre has organized a few national and international seminars and symposia till date. Besides, in the year 2012, the centre published the "M. C. Chaki Birth Centenary Commemoration Volume" (MCCBCCV).

\section{Concluding remarks}

Apart from the geometer of international fame in Professor M. C. Chaki, a closer view of the man himself is very nicely portrayed by his eminent student U. C. De, who was an Emeritus Professor of Pure Mathematics in Calcutta University. He wrote : 
Professor Chaki was a man of quiet and undemonstrative nature which lent him a certain aspect of austerity. Those who had not the privilege of coming in close contact with him were invariably inclined to regard him as a seriousminded puritan. In fact, the core of his personality consisted of a complex of sincerity, intellectual vigour and honesty, sympathetic comprehension and modesty. Those who had the privilege of coming in close contact with him discovered in him an innate, though restrained sense of humour, a love for literature and philosophy, an appreciation of music and an understanding of the weakness of human nature (NBCMS, 2008, pp. 9-10).

A man of high moral standing and humanistic outlook, Professor Chaki's memory will be cherished by his friends, colleagues, students and admirers not only for his academic eminence but also for his devoted and conscientious services for the development of science in our country. Besides, he will be remembered by the posterity for his significant contributions to the field of Differential Geometry.

\section{Acknowledgement}

The present writer expresses his deep sense of gratitude to his revered teacher Dr. Bandana Barua for going through the article and also for her valuable suggestions towards the improvement of the same. He also wishes to acknowledge with thanks the fruitful comments made by the learned referee on the article.

\section{Appendix}

\section{List of Publications of Professor M. C. Chaki}

1. Some formulas in Tensor calculus, Bull. Cal. Math. Soc., 42.4 (1950): 249-252.

2. On a non-symmetric harmonic space, Bull. Cal. Math. Soc., 44.1 (1952): 37-40.

3. (with H. Bagchi), Note on certain remarkable types of plane Collineations, Estr. dag. Ann. Scuola Norm. Super. di Pisa, Serie III, 6 (1952): 85-97.
4. (with H. Bagchi), Note on auto polar plane cubics, Rendi. Sem. Math. Uni. di Padova, 21 (1952): 316334.

5. (with H. Bagchi), Note on collineation-group connected with a plane quadrangle, Proc. Nat. Acad. Sci. India, 23.3, (1954).

6. On the line geometry of a curvature Tensor, Bull. Cal. Math. Soc., 47 (1955): 217- 226.

7. On a type of Tensor in a Riemannian space, Proc. Nat. Inst. Sci., India, 22A(2), (1956): 89-97.

8. Some formulas in a Riemannian space, Estr. dag. Ann. Scuola Norm. Super. di Pisa, Serie III, 10 (1956): 85-90.

9. Some theorems on recurrent and Ricci-recurrent spaces, Rendi. Sem. Math. Univ. di Padova, 26 (1956): 168-176.

10. (with B. Gupta), On conformally symmetric spaces, Ind. Jour. of Math., 5.2 (1963): 113-122.

11. (with R. N. Sen), On curvature restrictions of a certain kind of Conformally-flat Riemannian space of class one, Proc. Nat. Inst. Sci., India, 33A (1967): 100-102.

12. (with A. N. Raychowdhury), On Ricci-recurrent spaces of second order, Ind. Jour. of Math., 9 (1967): 279-287.

13. (with A. N. Raychowdhury), On conformally recurrent spaces of second order, Jour. Aust. Math. Soc., 10 (1969): 155-161.

14. (with D. Ghosh), On a type of K-contact Riemannian manifold, Jour. Aust. Math. Soc., 13.4 (1972): 447450.

15. (with D. Ghosh), On a type of Sasakian space, Jour. Aust. Math. Soc., 13.4 (1972): 508-510.

16. On conformally recurrent Kähler spaces, Tensor, N. S., 25, (1972): 179-182.

17. (with A. N. Raychowdhury) : On a type of Kähler space, Mathematica (Cluj), 16(39) 2 (1974): 223-227. 
18. (with A. K. Ray): On conformally flat generalized 2-Recurrent spaces, Publ. Math. Debrecen, 22.1-2, (1975): 95-99.

19. (with K. K. Sharma): A particular conformally symmetric space, Colloquium Mathematicum, 35.1 (1976): 87-90.

20. (with K. K. Sharma): Corrections to 'a Particular conformally symmetric space', Colloquium Mathematicum, 38.1 (1977): 169.

21. (with D. Ghosh) : On conformally 2-recurrent spaces, Mahavisva, 1.14, 29.1 (1977): 21-23.

22. (with A. K. Ray) : On certain types of Kähler spaces, Publ. Math. Debrecen, 26.3-4 (1979): 255-262.

23. (with A. Konar) : On a type of semi-symmetric connection on a Riemannian manifold, Jour. Pure Math., 1, (1981): 77-80.

24. (with U. C. De) : On a type of Riemannian Manifold with conservative conformal curvature tensor, Comptes Rendus Acad. Bulgare des Sciences, 34.7 (1981): 965-968.

25. (with U. C. De) : On a type of Riemannian manifold with conservative conformal curvature tensor, Annales de la Soc. Scient. de Bruxelles, 95.2 (1981): 81-84.

26. (with M. Tarafdar) : On a type of Sasakian manifold, Bull. Math. Soc. Sci. Math. de la R. S. de Roumanie, 27 (75) 3 (1983): 217-220.

27. (with S. K. Kar) : On a type of semi-symmetric connection on a Riemannian manifold, Comptes Rend. Acad. Bulgare des Sc., 36.1 (1983): 57-60.

28. (with G. Kumar) : On a type of semi-symmetric connection on a Riemannian manifold, Analele stiint. ale Univ. "AL I CUZA” din Iasi, 29.2 (1983): 41-44.

29. (with G. Kumar) : On semi-decomposable generalized projective 2-recurrent Riemannian spaces, Mathematica (Cluj), 26 (49) 1 (1984): 21-28.

30. (with S. K. Kar): On a type of semi-symmetric metric connection on a Riemannian manifold, Jour. Pure Math., 4, (1984): 102-107.
31. (with B. Chaki) : On a type of conformally flat nearly Kähler manifold, Analele stiint ale Univ. “ $A L$ I CUZA” din Iasi, 31.3 (1985): 235-238.

32. (with B. Chaki): On Pseudo-symmetric manifolds admitting a type of semi symmetric connection, Soochow Jour. Math., 13.1 (1987): 1-7.

33. On pseudo symmetric manifolds, Analele stiint. ale Univ. “AL I CUZA” din Iasi, 33.1 (1987): 53-58.

34. On pseudo Ricci symmetric manifolds, Bulg. Jour. Phys., 15.6 (1988): 526-531.

35. (with M. Tarafdar) : On conformally flat pseudoRicci symmetric manifolds, Periodica Mathematica Hungarica, 19.3 (1988): 209-215.

36. (with G. Kumar) : On semi-decomposable generalized conformally 2-recurrent Riemannian spaces, Mathematica (Cluj), 30(53).1 (1988): 11-18.

37. (with U. C. De) : On pseudo symmetric spaces, Acta Math. Hung., 54.3-4 (1989): 185-190.

38. (with S. K. Saha) : On pseudo projective symmetric manifolds, Bull. Inst. Math. Acad. Sinica, 17.1 (1989): 59-65.

39. (with M. Tarafdar) : On a type of Sasakian manifold, Soochow Jour. Math., 16.1, (1990): 23-28.

40. (with B. Barua) : On a new type of Riemannian manifold and its application to general relativity, Mahavisva, 4.1-2 (1991): 63-65.

41. (with P. Chakrabarti) : On Conformally Flat PseudoRicci Symmetric Manifolds, Tensor, N.S., 52.3(1993): 217-222.

42. (with G. Kumar) : On semi-decomposable generalized projective 2-recurrent Riemannian spaces, Univ. Nacl. Tucuman. Rev., Ser. A 30.1-2 (1993): 129-139.

43. On generalized pseudo symmetric manifolds, Publ. Math. Debrecen, 45.3-4 (1994): 305-312.

44. (with S. K. Saha): On pseudo projective Ricci symmetric manifolds, Bulg. Jour. Phys., 21.1-2 (1994): $1-7$. 
45. (with S. Koley) : On generalized pseudo Ricci symmetric manifolds, Periodica Mathematica Hungarica, 28.2 (1994): 123-129.

46. (with S. Koley) : On generalized pseudo projective Ricci symmetric manifolds, Analele stiint. ale Univ. “AL I CUZA" din Iasi, 41.1 (1995): 75-84.

47. (with S. Ray) : Space-times with covariant-constant energy-momentum tensor, Int. Jour. Theo. Phys., 35.5 (1996): 1027-1032.

48. (with M. L. Ghosh) : On quasi-confomally flat and quasi-conformally conservative Riemannian manifolds, Analele stiint. ale Univ. "AL I CUZA" din Iasi, 43.2 (1997): 375-381.

49. (with S. P. Mondal) : On generalized pseudo symmetric maniflods, Publ. Math. Debrecen, 51.1-2 (1997): 35-42.

50. (with R. K. Maity) : On totally umbilical hypersurfaces of a Conformally flat pseudo Ricci symmetric manifold, Tensor, N.S., 60.3 (1998): 254-257.

51. (with P. Chakrabarti) : On a type of Conformally flat hypersurface of a Euclidean manifold, Tensor, N.S., 61.1 (1999): 7-13.

52. (with R. Miron, B. Barua) : Symmetries of the Synge metric in the relativistic optics, Bull. Cal. Math. Soc., 92.3 (2000): 219-224.

53. (with M. L. Ghosh) : On quasi Einstein manifolds, Ind. Jour. Math., (B. N. Prasad Birth Centenary Commemoration Volume II), 42.2 (2000): 211-220.

54. (with R. K. Maity) : On quasi Einstein manifolds, Publ. Math. Debrecen, 57.3-4 (2000): 297-306.

55. On generalized quasi Einstein mainfolds, Publ. Math. Debrecen, 58.4 (2001): 683-691.

56. (with B. Barua) : Conformal symmetries of Synge metric in a generalized Lagrange space, Bull. Cal. Math. Soc., 94.4 (2002): 325-328.

57. (with P. K. Ghoshal) : On quasi Einstein pseudo Ricci symmetric manifolds, Jour. Pure Math., 18 (2002): 29-36.
58. (with P. K. Ghoshal) : Some global properties of quasi Einstein manifolds, Publ. Math. Debrecen, 63.4 (2003): 635-641.

59. (with S. Ray-Guha) : On a type of space-time of General Relativity, Tensor, N.S., 64.3 (2003): 227-231.

60. On super quasi Einstein manifolds, Publ. Math. Debrecen, 64.3-4 (2004): 481-488.

61. (with T. Kawaguchi) : On almost pseudo Ricci symmetric manifolds, Tensor, N.S., 68, (2007): 10-14.

62. On a type of fluid space-time admitting heat flux, Rev. Bull. Cal. Math. Soc., 16(2), (2008): 191-194.

\section{Other publications}

1. The Chhau dance of Seraikela, The Modern Student, December, 1938.

2. The Gold standard - its theoretical aspect, The Modern Student, July, 1939.

3. In Memoriam: Rabindra Nath Sen (1896-1974), Bull. Cal. Math. Soc., 67.4 (1975): 251-257.

4. On the Indian Zodiac, J. AASM Biophys. Res Cent Inaugural Volume, (1987): 1-7.

5. On an attempt in India to prove Euclid's fifth postulate, Jour. of the Asiatic Society, 30.1-4 (1988): 119127.

6. (with B. Chaki) : Axiomatic Kinship Theory, SYRI, 1.1 (1990): 3-8.

7. Syamadas Mukhopadhyay (1866-1937), Jour. Pure Math., 7 (1990): 59-65.

8. Bhupati Mohan Sen (1888-1978), Biographical Memoir, INSA, 15 (1991): 75-78.

9. On Statistical Manifolds, Tensor, N.S., 61.1 (1999): 14-17.

10. (with B. Chaki) : Some recent results of generalized Statistical manifolds, Presented at the Fifth International Triennial Calcutta Symposium on Probability and Statistics, Department of Statistics, Calcutta University, December, 28-31, 2003. 


\section{Text Books}

1. A Text Book of Tensor Calculus, Calcutta Publisher, Kolkata, First published, 1987. (used as a reference book in the University of Athens, Greece.)

2. A Text Book of Analytic Geometry, Calcutta Publisher, Kolkata, First published, vol. I, 1979 and vol. II, 1984.

3. A Text Book of Vector Analysis, Calcutta Publisher, Kolkata, First published, 1991.

4. (with N C Bose Majumder) A Text Book of Analytical Dynamics, Joydurga Library, Kolkata, First published, 1987.

5. A Text Book of Astronomy, Moulik Library, Kolkata.

6. (with S. Koley) : Uttar-Madhyamik Ganit, Vol. I \& Vol. II, New Book Stall, Kolkata, First published, 1993.

\section{Terminology of Mathematics}

1. Ganitik Paribhasa, Vol. I, West Bengal State Book Board, Kolkata, First published, June 1989.

2. Ganitik Paribhasa, Vol. II, West Bengal State Book Board, Kolkata, First published, December 2012.

\section{Bibliography}

[1] Barua B. A tribute to Professor M. C. Chaki, Tensor, N.S., 74 (2013): 212-215.

[2] Chaki M. C. Bhupati Mohan Sen (1888-1978), Biographical Memoirs of Fellows of the Indian National Science Academy, 15 (1991): 75-78.

[3] Chaki M. C. On a non-symmetric harmonic space, Bulletin of Calcutta Mathematical Society, 44.1 (1952): 37-40.

[4] Chaki M. C. and Gupta B. On conformally symmetric spaces, Indian Journal of Mathematics, 5.2 (1963): 113-122.

[5] Chaki M. C. On pseudo symmetric manifolds, Analele stiint. ale Univ. "AL I CUZA" din Iasi, 33.1 (1987): 53-58.
[6] Chaki M. C. and Maity R. K. On quasi Einstein manifolds, Publ. Math. Debrecen, 57.3-4 (2000): 297-306.

[7] Chaki M. C. and Kawaguchi T. On almost pseudo Ricci symmetric manifolds, Tensor, N.S., 68 (2007): $10-14$.

[8] Chaki M. C. and Ray S. Space-times with covariantconstant energy-momentum tensor, International Journal of Theoretical Physics 35.5 (1996):10271032.

[9] De U. C. Manindra Chandra Chaki (1913-2007), News Bulletin of Calcutta Mathematical Society, 31.4-6 (2008): 9-10.

[10] Guha Sarbari. Professor M. C. Chaki and M. C. Chaki Centre for Mathematics and Mathematical Sciences, Calcutta, FACTA UNIVERSITATIS, Series: Mechanics, Automatic Control and Robotics, 3.13 (2003): 779-780.

[11] Koley S. Geometrician Professor M. C. Chaki (in Bengali), Jnan-O-Bijnan, Bangiya Bijnan Parishad, Kolkata, 7 (2017): 376-381.

[12] Mukherji P. and Bhattacharjee M. Pioneer Mathematicians of Calcutta University, University of Calcutta, (2014): 90-96.

[13] M. C. Chaki Birth Centenary Commemoration Volume, M. C. Chaki Centre for Mathematics and Mathematical Sciences, Kolkata, 2012, pp.18-19, 79.

[14] Mathematical Reviews, U.S.A., (Sept. 1993): 4970. 